\title{
Pen Pressure Features for Writer-Independent On-line Handwriting Recognition Based on Substroke HMM
}

\author{
Mitsuru NAKAI, Takashi SUDO, Hiroshi SHIMODAIRA and Shigeki SAGAYAMA* \\ Graduate School of Information Science, \\ Japan Advanced Institute of Science and Technology \\ \{mit, sudotaka, sim\}@jaist.ac.jp \\ *Graduate School of Information Science and Technology, \\ The University of Tokyo \\ sagayama@il.t.u-tokyo.ac.jp
}

\begin{abstract}
This paper discusses the use of pen pressure as a feature in writer-independent on-line handwriting recognition. We propose two kinds of features related to pen pressure: one is the pressure representing pen ups and downs in a continuous manner; the other is the time-derivative of the pressure representing the temporal pattern of the pen pressure. Combining either of them with the existing feature (velocity vector), a 3-dimensional feature is composed for character recognition. Some techniques of interpolating the pen pressure during the pen-up interval is also proposed for a pre-processing purpose. Through experimental evaluation using 1,016 elementary Kanji characters compared with the baseline performance using velocity vector only, the additional use of pen pressure improved the performance from $97.5 \%$ to $98.1 \%$ for careful writings and from $91.1 \%$ to $93.1 \%$ for cursive writings.
\end{abstract}

\section{Introduction}

Handwriting recognition is a useful character input method to small PDAs (personal digital assistant), in which it is desirable to be able to input quickly like memorandum writing. In such an environment, we may write characters which are hard to read by appearance, since a stroke tends to overlap and a character sometimes lose its shape. Our proposed on-line handwriting recognition system based on substroke HMM[4] is robust to such overlaps of strokes by employing a velocity feature vector that is a difference between two consecutive pen positions. For the further improvement of the recognition accuracy, it is necessary to investigate the high-dimensional features that combined various information obtained from an input device.

Today, we can detect handwriting pressures on several pressure-sensitive tablets. The pen pressure information contains much individuality of writers[7] and it is often employed for writer identification[2]. However, for writerindependent handwriting recognition, pen pressure information is used only as a binary feature indicating penup/pen-down[3,6], since it is thought that the pen pres- sure pattern is not a discriminant feature for handwritten characters. In Japanese, the pen pressure pattern is only used in a few researches [5, 8]. In those researches, some normalizing technique is needed for removing writer's individuality from the pen pressure. Accordingly, their recognition processing is performed after a character is written. Here, we propose on-line pen pressure features for writerindependent handwriting recognition. That is, the pen pressure features are extracted by using short-time analysis and it does not need off-line normalization processing. Therefore, these features can be directly used for our HMM-based recognition system.

\section{Handwriting Recognition Based on Sub- stroke HMM with Pen Pressure}

\subsection{Extraction of Writing Velocity, Direction and Pressure Features}

The proposed system basically consists of input features, HMMs, dictionaries and a decoder as shown in Fig. 1. In this paper, we use pen positions $(x, y)$ and pen pressure (z) sampled at a certain interval from the pen tablet. Let $(d x, d y)$ be the difference between two consecutive pen position samples and $(r, \theta)$ be the primary feature vector, where $r$ means the velocity $\left(\sqrt{d x^{2}+d y^{2}}\right)$ and $\theta$ means the direction of the velocity vector[1].

In this paper, we propose two new features, the pen pressure itself and its time-derivative, and combine them with the existing velocity feature vector.

\subsubsection{Pen Pressure}

The pen pressure represents pen ups and downs in a continuous manner. In our conventional research, we did not use pen-up-down information whether the pen leaves (penup) or touches (pen-down) the tablet surface. Instead, a pen-up is probabilistically recognized by observing velocity feature vectors, where the feature $(d x, d y)$ during pen-up interval means comparatively large displacement vector from the pen-up position to the next pen-down position. On the 


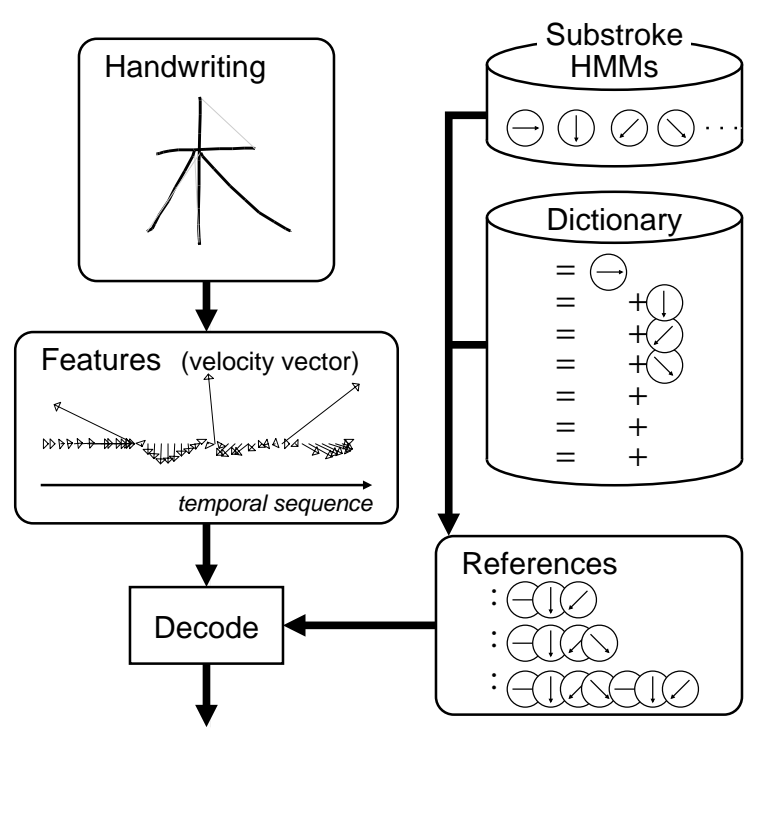

Figure 1. System configuration.

other hand, in this paper, the pen pressure $(z)$ is sampled at a certain interval and let the value itself be one of feature set. Also, a trajectory of the pen-up interval is sampled simultaneously; here we use a pen tablet that can detect pen positions even if the pen leaves the tablet surface about 8 $\mathrm{mm}$.

\subsubsection{Time-Derivative of Pen Pressure}

Although strength of the pen pressure is different in each writer, it is thought that the pattern of pen pressure fluctuation is common to many writers. Therefore, the pen pressure is regarded as the 3rd axis and we observe the pen trajectory $\left(x_{t}, y_{t}, z_{t}\right)$ in 3-dimensional space. Combining with the existing features, let $\left(r_{t}, \theta_{t}, \Delta z_{t}\right)$ be the feature vector, where $\Delta z_{t}$ is a regression coefficient of pen pressure and it is analyzed by $\sum_{i=-L}^{L} i z_{t+i} / \sum_{i=-L}^{L} i^{2}$. In the following sections, we call this feature a delta pressure.

\subsection{Substroke HMMs}

We model 25 substrokes of eight directions as shown in Fig. 2; eight long strokes ('A'-'H'), eight short strokes ('a'-'h'), eight pen-up movement ('1'-'8') and one pen-up-down movement ('0'). The HMMs of these substrokes have a topology of left-to-right model as shown in Fig. 3. The pen-down models have three states representing different stroke velocities, and pen-up models have only one state. It differs from the conventional models[4] that self-transition probability is added to pen-up model. Here, let $\lambda^{(k)}=\left(\boldsymbol{A}^{(k)}, \boldsymbol{B}^{(k)}, \boldsymbol{\pi}^{(k)}\right)$
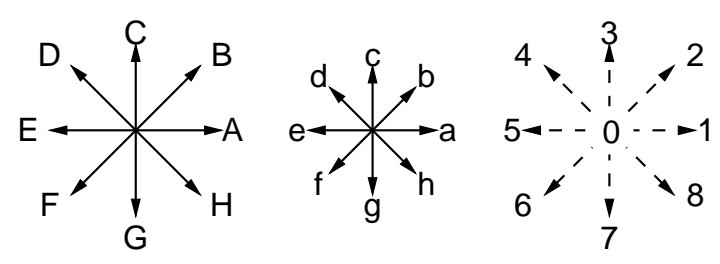

Figure 2. Substroke categories: $A-H(a-h)$ are long (short) substrokes with pen-down and 0-8 are the direction of pen-up.
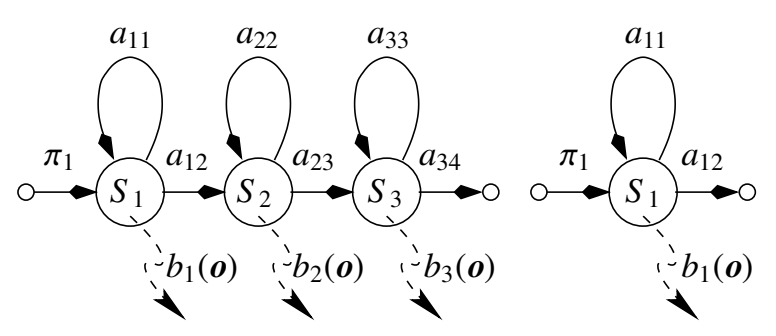

Figure 3. Substroke HMMs : (Left) pen-down model, (Right) pen-up model.

be the set of HMM parameters of substroke $k$, in which $\boldsymbol{A}^{(k)}=\left\{a_{i j}^{(k)}\right\} \quad:$ the state-transition probability distributions from state $S_{i}$ to $S_{j}$, $\boldsymbol{B}^{(k)}=\left\{b_{i}^{(k)}(\boldsymbol{o})\right\} \quad$ : the probability distributions of observation symbols $\boldsymbol{o}$ at state $S_{i}$, $\boldsymbol{\pi}^{(k)}=\left\{\pi_{i}^{(k)}\right\} \quad$ : the initial state probability distributions.

The observation probability distribution is represented by a $M$ mixtures of Gaussian distribution given by

$$
b_{i}(\boldsymbol{o})=\sum_{m=1}^{M} c_{i m} \frac{\exp \left(-\frac{1}{2}\left(\boldsymbol{o}-\boldsymbol{\mu}_{i m}\right)^{t} \boldsymbol{\Sigma}_{i m}^{-1}\left(\boldsymbol{o}-\boldsymbol{\mu}_{i m}\right)\right)}{\sqrt{(2 \pi)^{n}\left|\mathbf{\Sigma}_{i m}\right|}}
$$

with mean vector $\boldsymbol{\mu}$, covariance matrix $\boldsymbol{\Sigma}$ and weighting coefficient $c$. Here, the direction feature $(\theta)$ has a continuous probability distribution with $2 \pi$ cycle[1]. These model parameters can be trained by Viterbi training or Baum-Welch method.

\subsection{Recognition}

A decoder recognizes an input pattern by referring to the character's substroke sequence expanded from the hierarchical structure dictionary[4]. For example, the definition of the character " 二 " is 'a $6 A$ ' that represents two pendown strokes ' $a$ ' and ' $A$ ' are connected with pen-up model ' 6 ' in standard stroke order. Similarly, "子 " is 'A f 0 G d 4 $A$ ' and “字” is ' 55 g 3 A f 6 A f 0 G d 4 A', where “子” is the partial structure of "字” and those have a common 


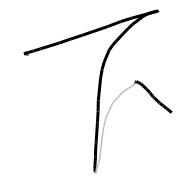

(a) Handwriting

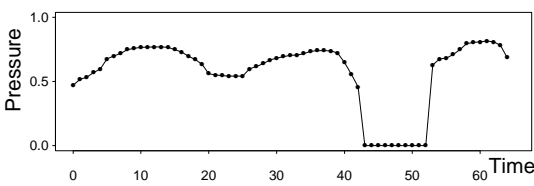

(b) Original pen pressure pattern

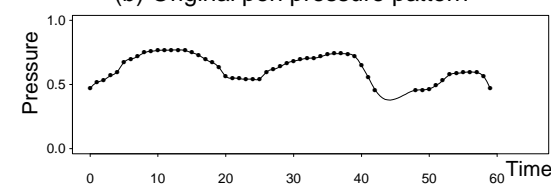

(c) Interpolated pattern by cubic splines

Figure 4. Cursive handwriting “下” and its interpolated pattern of the pen pressure.

substroke sequence. The decoder generates the connection model of each character from these character definitions and substroke HMMs, and then calculates the probability that an input pattern is produced from those HMMs by Viterbi search algorithm in a substroke network[4].

\section{Pre-processing of Feature Extraction for Cursive Writing}

The pen-up of handwriting can be probabilistically recognized in both case of using conventional velocity feature vector and using the pressure together. For example, even if we write "了" (two strokes) with one stroke or write “及” (three strokes) with two strokes, probably they can be recognized correctly. However, in extreme cursive writings, using the pressure information may conversely reduce the recognition accuracy, since the pressure characterizes penup-down and a pen-up may sometimes disappear by writing two or more strokes with one drawing. To overcome this problem, we propose a technique of interpolating the pen pressure information on the interval that carried out the pen-up correctly. Fig. 4 is an example of the cursive writing “下” (three strokes) and the 1st stroke and the 2nd stroke are drawn continuously (connected-stroke). Fig. 4(c) shows the result of approximating the pressure value of the pen-up interval to the fall-rise pattern of the connected-stroke, so that we can train only one substroke HMM per pen-up label. The interpolation algorithm is as follows. Let $t_{1}$ be a time when the pen leaves tablet and $t_{2}$ be a beginning time of next pen-down. At first, we set a pen pressure difference between $z_{t_{1}}$ and $z_{t_{2}}$ to 0.0 by adding a value $z_{t_{2}}-z_{t_{1}}$ to the pressure $z_{t}\left(t>t_{2}\right)$. Next, using the cubic splines method, $S$ points in the pen-up interval is interpolated. Here, the pen positions $(x, y)$ during the pen-up are also interpolated so that the velocity feature vector may become constant.

\section{Experimental Evaluation}

Handwriting database used in this evaluation is the JAIST IIPL (Japan Advanced Institute of Science and Tech-
Table 1. Comparison of features in careful handwriting recognition

\begin{tabular}{|c||c|c|c|c|c|}
\hline \multirow{2}{*}{ Features } & \multicolumn{4}{|c|}{$N$-best accumulative recognition rate (\%) } \\
\cline { 2 - 6 } & 1 & $\sim 2$ & $\sim 3$ & $\sim 5$ & $\sim 10$ \\
\hline
\end{tabular}

(a) with pen-up trajectories

\begin{tabular}{|l||l|l|l|l|l|}
\hline$r, \theta$ & 94.81 & 97.69 & 98.53 & 99.01 & 99.36 \\
\hline$r, \theta, z$ & 98.08 & 99.53 & 99.71 & 99.77 & 99.83 \\
\hline$r, \theta, \Delta z$ & 96.14 & 98.41 & 99.02 & 99.28 & 99.57 \\
\hline
\end{tabular}

(b) without pen-up trajectories

\begin{tabular}{|l||l|l|l|l|l|}
\hline$r, \theta$ & 97.47 & 99.23 & 99.60 & 99.77 & 99.87 \\
\hline$r, \theta, z$ & 97.98 & 99.56 & 99.78 & 99.84 & 99.89 \\
\hline$r, \theta, \Delta z$ & 97.54 & 99.27 & 99.63 & 99.80 & 99.87 \\
\hline
\end{tabular}

nology, Intelligence Information Processing Laboratory) database that consists of several kinds of data sets. Among them, we used two kinds of dataset written with standard stroke order; one was the careful writing dataset ( $\gamma$ set) with right stroke-count and the other was cursive writing dataset ( $\epsilon$ set). Both datasets cover 1,016 Japanese characters of old and new educational Kanji.

\subsection{Experiment 1: Careful Writing Recognition with Pen Pressure}

An experimental comparison of features was carried out on the 1,016 Japanese educational Kanji recognition task. The odd-numbered 30 writers from $\gamma$ dataset were used for estimating the HMM parameters by Viterbi training method, and the remaining even-numbered 30 writers were used for test. Additionally, we also compared two cases about whether pen-up trajectories were used or not. The HMMs were trained as 2 mixture Gaussian distributions.

The recognition results according to the feature sets are shown in Table 1. Generally, the recognition rates become high when the pen pressure information is used. About the trajectory of the pen-up interval, the recognition rate is improved only when it is used together with the pressure feature $(z)$.

\subsection{Experiment 2: Cursive Writing Recognition with Pen-up Interpolation}

From the $\epsilon$ dataset, the odd-numbered 34 writers were used for training HMMs, and the remaining even-numbered 34 writers were used for test. The other experimental conditions were the same as Experiment 1. However, the evaluation was carried out with or without the delta pressure feature $(\Delta z)$. It is thought that the pressure feature $(z)$ is unsuitable to use in the cursive writing recognition, where strong pen pressure is observed during pen-up interval of connected-stroke.

Fig. 5 shows the recognition rates when varying the number of interpolated points $S$ from 1 to 8 . In $S \geq 3$, the delta 


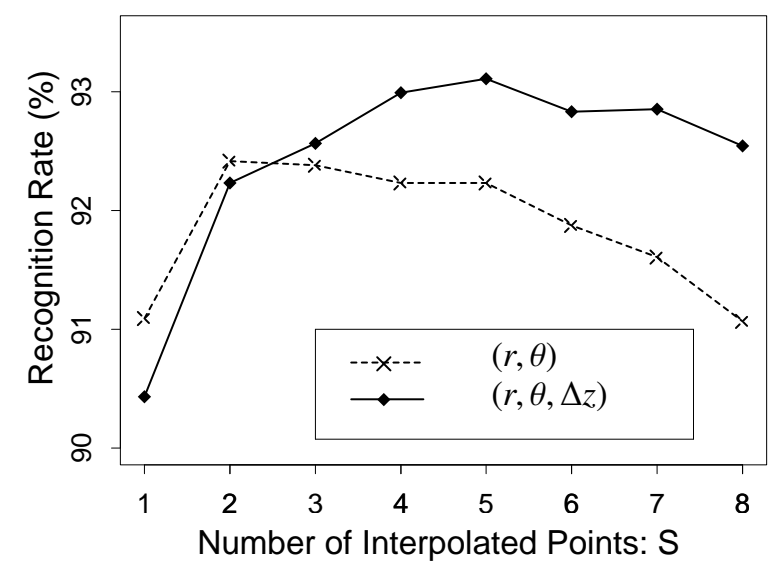

Figure 5. Recognition rate with the number of
interpolated points.

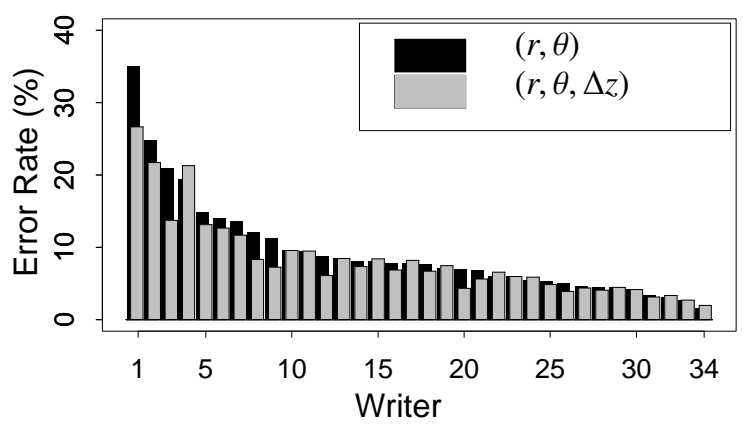

Figure 6. Error rate reduction for each writer.

pressure feature was effective in improving the recognition accuracy. As a result of training pen-up HMMs by using samples written with only one drawing, the self-transition probabilities of pen-up models were from 0.7 to 0.85 . Then, the expected number of observed point in a pen-up interval was from 3 to 6 . In correspondence to that, the recognition rate became the maximum in the case of $S=5$.

The recognition rate for each writer is shown in Fig. 6. We can see that a writer-independent model with the delta pressure was effective to many writers; the recognition rate was improved for 19 writers. This result means that the pattern of pen pressure fluctuation is common to many writers.

The improved sample of handwritings are shown in Fig. 7. Conversely, the samples that have changed to incorrect recognition are shown in Fig. 8. Here, “団 $\rightarrow$ 因” means that the recognition result changed from " 団” to “因” by using the delta pressure features. Totally, the number of improvement was 1,029 writings and the number of incorrectly changed characters was 89 writings.

Incidentally, the recognition rate of careful writing recognition is also improved to $97.91 \%$. It turns out that the effectiveness of the pen-up interpolation does not depend on the quality of handwritings.

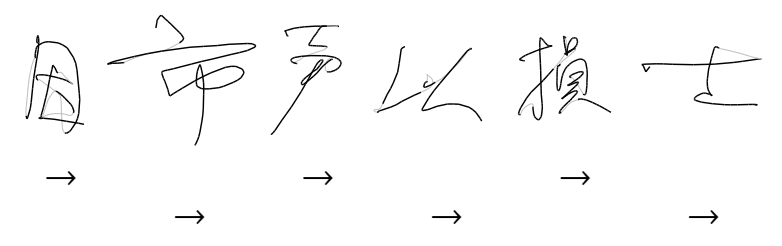

Figure 7. Improved samples by using $\Delta$ pressure feature.

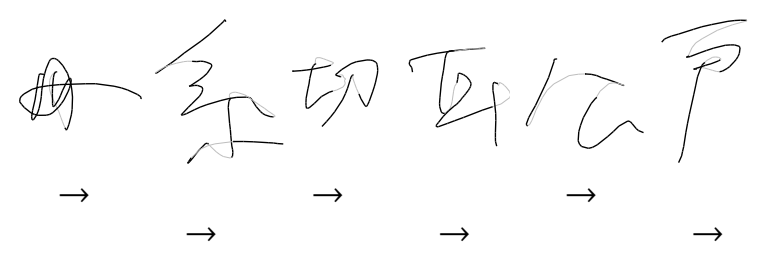

Figure 8. Error samples by using $\Delta$ pressure feature.

\section{Conclusion}

We have proposed on-line pen pressure features for writer-independent handwriting recognition and shown its validity through experiments. Furthermore, the preprocessing technique for handwritings has been proposed, and improvement in the recognition rate was achieved in both of cursive writings and the careful writings. We expect that the recognition accuracy of cursive writings would be improved further by combining substroke contextdependent model.

\section{References}

[1] N. Akira, M. Nakai, H. Shimodaira, and S. Sagayama. "An Investigation into the Representation of Feature Vectors and their Stochastic Modeling by Stroke-based HMM for On-line Handwriting Character Recognition" (in Japanese). Technical report of IEICE, PRMU2000-134:31-38, Dec. 2000.

[2] M. Kikuchi and N. Akamatsu. "Development of Speedy and High Sensitive Pen System for Writing Pressure and Writer Identification". Proc. ICDAR'01, pages 1040-1044, Sept. 2001.

[3] A. Kosmala, J. Rottland, and G. Rigoll. "An Investigation of the Use of Trigraphs for Large Vocabulary Cursive Handwriting Recognition". Proc. ICASSP'97, 4:3373-3376, Apr. 1997.

[4] M. Nakai, N. Akira, H. Shimodaira, and S. Sagayama. "Substroke Approach to HMM-based On-line Kanji Handwriting Recognition". Proc. ICDAR'01, pages 491-495, Sept. 2001.

[5] Y. Sato and H. Adachi. "Online Recognition of Cursive Writings" (in Japanese). IEICE Trans. (D), J68-D(12):2116-2122, Dec. 1985.

[6] K. Takahashi, H. Yasuda, and T. Matsumoto. "A Fast HMM Algorithm for On-line Handwritten Character Recognition". Proc. ICDAR'97, 1:369-375, Aug. 1997.

[7] Y. Yamazaki, Y. Mizutani, and N. Komatsu. "Extraction of Personal Features from Stroke Shape, Writing Pressure and Pen Inclination in Ordinary Characters". Proc. ICDAR'99, pages 426-429, Sept. 1999.

[8] P. Zhao, Y. Sato, and M. Yoshimura. "Generating Generic Reference for Cursive Wrirings" (in Japanese). IPSJ Trans., 34(3):418-425, Mar. 1993. 